Mula, M. \& Trimble, M. R. (2009) Music and madness: neuropsychiatric aspects of music. Clinical Medicine, 9, 83-86.

Pasternak, C. (2007) What Makes Us Human. One World.

Stewart, L., von Kriegstein, K. \& Warren, J. D. (2006) Music and the brain: disorders of musical listening. Brain, 129, 2533-2553.
Thaut, M. H. (2005) The future of music in therapy and medicine. Annals of the New York Academy of Science, 1060, 303-308.

Trimble, M. R. (2007) The Soul in the Brain: The Cerebral Basis of Language, Art and Belief. Johns Hopkins University Press.

Trimble, M. R. (2012) Why Humans Like to Cry. Tragedy, Evolution and the Brain. Oxford University Press.

\section{THEMATIC} PAPER

\title{
Music therapy for mental disorder and mental health: the untapped potential of Indian classical music
}

\author{
Shantala Hegde
}

Neuropsychology Unit and Cognitive Psychology and Cognitive Neurosciences Laboratory, Department of Clinical Psychology, National Institute of Mental Health and Neurosciences, Bangalore, India, email shantala.hegde@gmail. com

\begin{abstract}
Music is a universal human trait. The healing power of music has been acknowledged in almost all traditions of music. Music therapy is moving from a social-science model focusing on overall health and well-being towards a neuroscience model focusing on specific elements of music and its effect on sensorimotor, language and cognitive functions. The handful of evidence-based music therapy studies on psychiatric conditions have shown promising results. Traditional music, such as Indian classical music, has only recently been evaluated in evidence-based research into music therapy. The need for systematic research in this area is underscored.
\end{abstract}

\section{An overview of Indian classical music}

Music, like language, is a ubiquitous human trait. Like any other art form, music has always been viewed from an aesthetic perspective and its healing power has been documented in various traditions of music across the world. Indian classical music (ICM) is perhaps one of the oldest forms of music. It dates back to the ancient scriptures of India, the Vedas (dated around 5000-2000 BC), and is said to have originated from one of the four Vedas, the Samaveda. The Vedas consist of verses in Sanskrit for chanting. The two present forms of ICM, known as north Indian classical music (or Hindustani classical music) (NICM) and south Indian classical music (or Carnatic classical music) branched out from the same tradition around the 13th century AD. Ragas (from the Sanskrit phrase ranjayiti iti ragaha), meaning 'one which induces emotion in the mind', and taal (a rhythmic structure and cycle) form the core of ICM. The ragas provide the framework for melodic elaboration and are defined as a melodic basis for compositions and improvisations (Jairazbhoy, 1995). There are hundreds of ragas in these two traditions. Each raga has specific notes (swar) for ascending and descending, and a specific manner in which the notes are combined. Certain notes in the ragas are considered crucial. The most important note is called the vadi-swar and the second most important note is called the samvadi-swar. Each raga has a unique melodic line, like a signature tune or motif, which is called the pakkad. Each raga is associated with a specific affective theme (rasa or ras, a Sanskrit word meaning 'the essence'). Ragas are said to evoke one or more of these emotions (sadness, romance, peace, strength/courage, anger, devotion, longing, passion).

Expression of the raga-rasa aspect is considered the primary goal in ICM, and this expression is intended to vary dynamically during performance. The presentation of the raga in ICM therefore evolves over time, in various stages (alap, jhor-jala, gat, vilambit, dhrut) and dimensions. Variations are introduced not only in the melodic improvisation, but also in the composition, tempo and complex rhythmic cycle.

A study investigating the variations in emotional experience during the different phases of raga elaboration found that emotional variations within certain ragas were often larger than between ragas. Indian classical musicians have the ability to strongly vary the expressivity associated with a specific raga in their performances, but within the constraints of the raga framework (Hegde et al, 2012).

In an electroencephalography (EEG) study, 20 musically untrained individuals listened to NICM ragas; they showed increased overall alpha, delta and theta power in comparison with an eyes-closed rest condition. The observed changes during music listening had previously been linked with highly relaxed states, such as meditative states (Hegde $e t$ al, 2012). Listening to certain ragas, for example 'Desi-todi', for 30 minutes every day for 20 days has been shown to produce a significant decrease in systolic and diastolic blood pressure, to reduce stress, anxiety and depression, and to enhance feelings of life satisfaction, experience of hope and optimism. Changes in heart rate were much more evident in females than males (Gupta \& Gupta, 
2016). Ragas are considered to have healing powers and the ability to improve overall health.

\section{Music therapy and the journey from a social to a neuroscience perspective}

Using music as a therapy dates back to prehistoric times. It has been predominantly driven by the recreational and social-science model, focusing on well-being and emotional health. The World Federation of Music (1996) defines music therapy as follows:

the use of music and its elements by a qualified music therapist with a client or group, in a process designed to facilitate and promote communication, relationships, learning, mobilization, expression, organization and other relevant therapeutic objectives, in order to meet physical, emotional, cognitive and social needs. Music therapy aims to develop potentials and/or restore functions of the individual so that he or she can achieve better intraand inter-personal integration and, consequently, a better quality life through prevention, rehabilitation or treatment. (Cited in Bruscia, 2000, p. 286)

Over the past three decades, music has been studied as a cognitive phenomenon. Music is seen as a complex set of different processes and characteristics such as pitch, pitch interval, tempo, rhythm, melodic contour and rhythmic contour. Scientific evidence has been produced pertaining to various clinical conditions, and to musically trained and untrained individuals. This field of research has emerged as a specialised branch of cognitive neuroscience and psychology, and is variously referred to as neuromusicology, music cognition or music psychology. Advances in research methods, tools and techniques, such as functional magnetic resonance imaging (fMRI), EEG, event-related potential (ERP), transcranial magnetic stimulation (TMS) and magnetic encephalography (MEG), have contributed to the exponential growth of this field. Improved understanding of the neural correlates of music has in turn contributed significantly to the understanding of the functioning of the human brain and has facilitated a paradigm shift in the field of music therapy. Music is considered to be a biological phenomenon and not just a sociocultural phenomenon. This has led to a shift from social science and interpretive models to neuroscience-based music therapy, targeting multiple domains of functioning (Hegde, 2014).

\section{Music therapy: an apt method of treatment in psychiatric conditions}

Research on music and its effect on brain functions has produced insights into the nature of the brain, especially the phenomenon of 'neural plasticity'. Highly trained musicians are considered an ideal population with whom to study neural plasticity. Musical performance engages a host of cognitive functions, including information processing, attention, language, memory functions and executive functions. Engaging with music is known to have benefits for the cognitive, emotional, physiological as well as social well-being of an individual (Zatorre, 2005). A recent case study reported that a professional musician with a long history of refractory epilepsy did not show any cognitive deficits nor deficits in musical skills before and after temporal lobectomy. The authors suggest that such a preservation of cognitive and musical skills is due to cognitive reserve, achieved through the long duration of musical training (Hegde $e t$ al, 2016). In a study of patients with Parkinson's disease, a high correlation was found between cognitive functions and performance of various temporal components of rhythm such as tempo, beat discrimination and beat perception in musical context (Biswas et al, 2016).

Music is a strong elicitor of emotion but also facilitates regulation of emotion. Music activates the cortical network associated with emotion, including the frontal system, which is involved in emotion processing. Music is known to engage brain areas such as the mesolimbic area and the nucleus accumbens, the reward centre of the brain, which is known to be involved in real-life emotional experiences. Music alters psychophysiological parameters such as pain perception, relaxation, blood pressure, and respiration and heart rate.

Neurochemical changes due to music can be viewed in four different domains (Chanda \& Levitin, 2013):

- reward, motivation and pleasure, mediated by dopamine and opioids

- stress and arousal, mediated by cortisol, corticotrophin-releasing hormone $(\mathrm{CRH})$ and adrenocorticotrophic hormone (ACTH)

- immunity, mediated by serotonin and the peptide derivatives of proopiomelanocortin (POMC), alpha-melanocyte-stimulating hormone and beta-endorphin

- social affiliation, mediated by oxytocin.

Music therefore is an apt intervention to address multiple domains, such as cognitive, emotional, social domains of functioning, which are adversely affected in psychiatric conditions.

Systematic, randomised controlled studies of music therapy in psychiatric conditions have recently been carried out. Cochrane reviews of its use in patients with depression (Maratos et al, 2008) and people with schizophrenia and schizophrenia-like illnesses (Mossler et al, 2011) have reported that the intervention improves mood and targets psychopathology. These reviews also report that patients accept and participate in music therapy; the drop-out rates are low, which suggests that music has the ability to sustain the interest of patients. Deficits in prosody, musical emotion, rhythmic contour and incidental musical memory have been recently reported in patients with schizophrenia. Melody and rhythm perception, and musical memory, are known to predict verbal working memory, verbal memory and facial emotion recognition in patients with schizophrenia (Chandrashekaran, 2015). Listening to music has also been shown to have beneficial effects in improving mood and certain cognitive functions in clinical conditions such as depression, stroke and 
dementia. Although real sadness is avoided, sad music is often listened to keenly. In a seminal work, it was reported that listening to sad music led to four different types of reward, relating to imagination, emotion regulation, empathy and lack of 'real-life' implications (Taruffi \& Koelsch, 2014).

\section{Conclusion and future directions}

The present article provides only a bird's eye view of the vast subject, which has grown exponentially in the recent past and is continuing to do so. Musical training and evidence-based music therapy improve sensorimotor, language and cognitive functions in the non-musical domain. So far, the Western classical music tradition has been scientifically examined to a far greater extent than music from any other tradition. The findings are generalisable, however, as different forms of music from various cultures have certain common features. Nonetheless, traditional music of various cultures should be systematically studied to determine their unique value, if any, in a therapeutic scenario. Scientific investigations of the innumerable ragas of ICM are still in their infancy. Systematic studies on various psychiatric conditions are imperative.

\section{References}

Biswas, A., Hegde, S., Jhunjhunwala, K., et al (2016) Two sides of the same coin: impairment in perception of temporal components of rhythm and cognitive functions in Parkinson's disease. Basal Ganglia, 6, 63-70.
Bruscia, K. (2000) Definindo Musicoterapia [Defining Music Therapy]. Enelivros.

Chanda, M. L. \& Levitin, D. J. (2013) The neurochemistry of music Trends in Cognitive Sciences, 17, 179-193.

Chandrashekaran, N. (2015) Musical Deficits in Schizophrenia - Its Relation with Deficits in Cognition and Emotion Recognition. MPhil thesis in clinical psychology, National Institute of Mental Health and Neurosciences, Bangalore, India

Gupta, U. \& Gupta, B. S. (2016) Gender differences in psychophysiological responses to music listening. Music and Medicine, 8, 53-64

Hegde, S. (2014) Music-based cognitive remediation therapy for patients with traumatic brain injury. Frontiers in Neurology, 5, 34

Hegde, S., Aucouturier, J.-J., Ramanujam, B., et al (2012) Variations in emotional experience during phases of elaboration of north Indian raga performance. In 12th International Conference on Music Perception and Cognition and the 8th Triennial Conference of the Cognitive Sciences of Music, July 23-28 2012 (eds E. Cambourpoulos, et al), pp. 412-413.

Hegde, S., Bharath, R. D., Rao, M. B., et al (2016) Preservation of cognitive and musical abilities of a musician following surgery for chronic drug-resistant temporal lobe epilepsy: a case report. Neurocase, 22, 1-6.

Jairazbhoy, N. A. (1995) The Ragas of North Indian Music: Their Structure and Evolution. Popular Prakashan Pvt Ltd.

Maratos, A., Gold, C., Wang, X., et al (2008) Music Therapy for Depression. Cochrane Library.

Mossler, K., Chen, X., Heldal, T. O., et al (2011) Music therapy for people with schizophrenia and schizophrenia-like disorders. Cochrane Database of Systematic Reviews, (2), Cd004025.

Taruffi, L. \& Koelsch, S. (2014) The paradox of music-evoked sadness: an online survey. Plos One, 9, E110490.

Zatorre, R. (2005) Music, the food of neuroscience? Nature, 434 312-315.

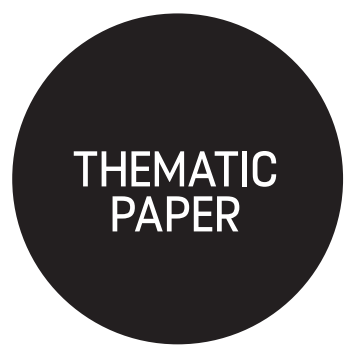

\title{
Music performance anxiety in classical musicians - what we know about what works
}

\author{
Raluca Matei ${ }^{1}$ and Jane Ginsborg ${ }^{2}$
}

${ }^{1}$ Royal Northern College of Music, Manchester, UK, email raluca. matei@student rncm.ac.uk

2Royal Northern College of Music Manchester, UK, email jane. ginsborg@rncm.ac.uk
When pursued professionally, the demands of musical training and performance can interfere with musicians' well-being and health. Music performance anxiety, while energising at optimal levels, impairs performance quality when excessive. A range of interventions has been explored to address it. However, the poor methodological quality of such studies and the complexity of this issue should mobilise further research resources in this direction.

\section{Music performance anxiety}

Might the mere presence of an audience be enough to turn music making, so often therapeutic, into an anxiety-enhancing activity? Fancourt et al (2015) found higher levels of glucocorticoids (cortisol and cortisone) in singers performing in public than when they were singing without an audience. Furthermore, research using a virtual reality performance simulator demonstrated that the audience does not even have to be 'real' for anxiety to be triggered or heart rate raised (Aufegger et $a l, 2016)$.

Those who succeed in entering the highly competitive field of classical music must not only possess personal attributes such as determination and resilience but also acquire cognitive and social skills, instrument-specific motor skills, coping skills adequate to the psychological demands of public performance, and the ability to manage their time and be responsible for their physical and mental health. While musicians report the highest level of job satisfaction, they are also among the five occupational groups most likely to report mental illness (Brodsky, 1996). 